\title{
PERCEPÇÃO DA AUTOIMAGEM CORPORAL EM DEPENDENTES DE CRACK
}

\section{Alexandre Dido Balbinot}

Educador físico. Mestre em saúde coletiva. Docente especialista do Centro de Atendimento Educacional Especializado do Município de Cachoeirinha (RS) Brasil.

\section{Renata Brasil Araújo}

Psicóloga. Doutora em psicologia. Coordenadora dos Programas de Dependência Química e Terapia Cognitivo-Comportamental do Hospital Psiquiátrico São Pedro. Porto Alegre (RS) Brasil.
RESUMO: Este estudo objetivou analisar a imagem corporal, a composição corporal e fatores associados em dependentes de crack internados. Trata-se de um estudo quantitativo com delineamento transversal composto por 100 sujeitos hospitalizados para desintoxicação. Para a coleta de dados foram utilizados como instrumentos: ficha de dados sociodemográficos; escala de Silhuetas e avaliação antropométrica. Observou-se Índice de Massa Corporal médio de $24,01 \mathrm{~kg} / \mathrm{m}^{2}( \pm 3,65)$, distorção da imagem de $-1,48( \pm 1,80)$, e insatisfação corporal de $2,13( \pm 2,85)$. A análise estatística mostrou diferença significativa entre a imagem da silhueta real e a imagem da silhueta atual dos sujeitos $\left(\chi^{2}=153,25 ; p<0,001\right)$. Concluiu-se que existe diferença entre a imagem da silhueta que os indivíduos possuem de si mesmo, quando comparada com aquela que se equipara ao seu IMC, mais que isto ficou evidente a existência de insatisfação dos sujeitos quanto às suas silhuetas, assim como o desejo que seu tamanho corporal fosse aumentado.

PALAVRAS-CHAVE: Imagem corporal; Cocaína crack; Antropometria.

\section{PERCEPTION OF BODY SELF-IMAGE IN CRACK- DEPENDENT PEOPLE}

ABSTRACT: Current qualitative study with transversal design analyzes body image, body composition and factors associated with 100 crackdependent people, hospitalized for de-intoxication. Data collection comprised: a card with social and demographic data; silhouette scales and anthropometric evaluation. Mean Body Mass Index was $24.01 \mathrm{~kg} /$ $\mathrm{m}^{2}( \pm 3.65)$; image distortion $-1.48( \pm 1.80)$ and body dissatisfaction $2.13( \pm 2.85)$. Statistical analysis revealed a significant difference between the image of true silhouette and the image of the subjects' current silhouette $\left(\chi^{2}=153.25 ; \mathrm{p}<0.001\right)$. Results show that differences exist between the silhouette image people have of themselves when compared to that equivalent to their BMI. The subjects were dissatisfied by their silhouette and desired that their body size would be increased.

KEY WORDS: Body image; Cocaine crack; Anthropometry.

\section{INTRODUÇÃO}

A preocupação com a imagem corporal não é algo recente em

Autor correspondente:

Alexandre Dido Balbinot

E-mail: adbalbinot@gmail.com nossa sociedade, mas acompanha a humanidade e vem modificando seus padrões corporais ao longo do tempo. Esta imagem está relacionada com a identidade do sujeito assim como ao nível de satisfação 
pessoal, podendo desenvolver bem-estar, mas também fomentar sentimento oposto e ocasionar uma série de patologias. $^{1-4}$

A imagem corporal é um importante componente do mecanismo de identidade pessoal que se apresenta como a representação interna da estrutura corporal, com uma construção multidimensional que define amplamente a aparência física pessoal. Ela é multifacetada e passível de modificações, sendo constituída pelo contínuo intercâmbio entre inúmeros fatores, tanto físicos e psíquicos, quanto sociais. ${ }^{5-9}$.

Entre os fatores físicos é possível destacar, principalmente, a relevância do somatotipo e dos componentes implícitos a este, como altura, peso e perímetros corporais. Entretanto, a questão da saúde e bem-estar pessoal, que não se restringe somente à ausência de patologias físicas e metabólicas, é outro aspecto importante a ser ressaltado. ${ }^{1,6,7,10}$

Também podem ser observados fatores relacionados aos aspectos sociais, decorrentes de uma tendência cultural, pois a sociedade molda e transmite, principalmente por meio da mídia, sua concepção de corpo ideal. É observado, na sociedade atual, o culto à magreza entre as mulheres e um culto à força e corpo volumoso entre os homens. ${ }^{3-6,8-10}$

Este corpo ideal da cultura atual, muitas vezes, não está ao alcance do indivíduo e tende emergir a insatisfação com o corpo, proveniente da distorção da percepção, comportamento ou cognição em relação ao próprio corpo, gerando desconforto e aumento da preocupação com o mesmo. ${ }^{11}$ Pesquisas têm demonstrado que o distanciamento entre a autoimagem atual do indivíduo e aquela considerada ideal fomenta o desenvolvimento de sofrimento em nível psíquico. ${ }^{1,5,6,9,12,13}$

Estudos em Melbourne, na Austrália, observaram que a grande preocupação com a imagem corporal está relacionada com o surgimento de sintomas de ansiedade, desordens alimentares e quadros depressivos em adolescentes. ${ }^{1}$ Os resultados relatados por Melbourne se assemelharam àqueles encontrados em estudo com adultos do sul do Brasil ${ }^{11}$, e de estudo americano13 com homossexuais do sexo masculino, em que foram encontradas associações entre a insatisfação corporal quando comparada com depressão e desordens alimentares.

Muitas vezes existe uma diferença significativa entre a imagem corporal que a pessoa refere sobre sua a percepção da própria imagem corpora, o que considera sendo o ideal ou o que preconiza para a sua vida em relação à saúde e estética. A discrepância da forma corporal atual e a ideal são iguais ao nível de insatisfação. Segundo a literatura, as mulheres apresentam maior nível de insatisfação corporal do que os homens. ${ }^{5-7}$

A escala de silhuetas constitui um importante instrumento para avaliar percepção da imagem corporal subjetiva dos indivíduos, assim como aquilo que o sujeito considera como corpo ideal e que almeja para si. Por meio desse instrumento, é possível quantificar a (in) satisfação dos indivíduos frente a sua imagem corporal, o que, como referido anteriormente, é preditor de bem -estar. ${ }^{2,8}$

Em contrapartida ao bem-estar, assim como a insatisfação corporal a intoxicação desencadeada pelo consumo de crack pode produzir quadros de transtornos alimentares, e, a partir do uso de crack, desencadear o surgimento de transtornos paranoides severos. ${ }^{14,15}$ Nesta perspectiva é descrito, em estudo realizado no sul do Brasil16, que o Índice de Massa Corporal (IMC - médio) de dependentes de crack ao ingressarem em internação hospitalar para desintoxicação é inferior ao observado em estudo antropométrico contendo amostras da população geral. ${ }^{17}$

Esta característica proveniente da população usuária de crack sugere a possibilidade de insatisfação em relação a sua própria imagem e consequente carga de sofrimento, podendo ser estes fatores gerador de lapso e/ ou recaída. Em consonância a esta concepção, estudo de brasileiro, realizado com adolescentes, demonstrou associação estatisticamente significativa entre a insatisfação com a imagem corporal e o consumo de tabaco. ${ }^{18}$

Semelhantemente, estudo composto por usuários de tabaco evidenciou que a preocupação com a imagem corporal em sujeitos abstinentes de tabaco pode influenciar as decisões acerca da manutenção da abstinência, demonstrando que aqueles que relatam insatisfação com a imagem corporal possuem até 3,4 vezes mais chance de desencadear lapso e consequentemente a recaída. ${ }^{19}$

Estes estudos vão ao encontro do pensamento de Sengik e Scortegagna que refletem em sua obra a questão da insatisfação corporal que está atrelada às angústias e ansiedades nos sujeitos, e que tendem a culminar na utilização de substâncias psicoativas como uma estratégia 
de refúgio ${ }^{20}$ Assim, o presente trabalho teve por objetivo analisar a imagem corporal e a avaliação antropométrica em dependentes de cocaína fumada/crack internados para desintoxicação.

\section{METODOLOGIA}

Trata-se de uma pesquisa quantitativa de cunho observacional com delineamento transversal e amostra não aleatória composta por 100 sujeitos do sexo masculino em tratamento na modalidade de internação hospitalar pela dependência de crack. Teve-se como critério de inclusão no presente estudo: possuir dependência de cocaína/crack pela CID-10; possuir idade entre 18 e 60 anos; ter o crack como droga de preferência. Os critérios de exclusão no presente estudo foram: diagnóstico de retardo mental comprovado por laudo; analfabetismo; condição que prejudicasse o desempenho do indivíduo para responder à pesquisa (alteração do estado de consciência). ${ }^{21}$

Os dados foram coletados nos primeiros sete dias, a contar do momento de ingresso ao atendimento, e para coleta de dados utilizaram-se os seguintes instrumentos:

Ficha de dados sociodemográficos e perfil de uso de substâncias psicoativas: ficha de dados sociodemográficos elaborada para definir o perfil da amostra estudada, identificando características que pudessem ser importantes para avaliar os sujeitos e verificação dos dados referentes ao consumo das substâncias psicoativas.

Avaliação antropométrica: os dados antropométricos mensurados foram os seguintes: - peso (mensurado em kg, com intervalo de medida de $0.050 \mathrm{~kg}$ ); estatura (utilizando a medida metros (m), com intervalo de medida de $0,5 \mathrm{~cm}$ ); - perímetro de cintura e quadril (mensurado em centímetros, com intervalo de medida de $0,1 \mathrm{~cm})$;

Escala de silhuetas2: instrumento que avalia componentes da imagem corporal, para utilização em pesquisas clínicas e epidemiológicas. É composto por 15 cartões que apresentam figuras representativas de diferentes intervalos crescentes de Índice de Massa Corporal (IMC). Cada cartão possui uma imagem de silhueta corporal que corresponde a uma média e um intervalo de
IMC real para possível classificação dos indivíduos avaliados. Durante a aplicação do protocolo, utilizando-se os cartões da Escala de Silhuetas dispostos de forma crescente, era perguntado ao indivíduo na seguinte ordem: "Qual figura representa o seu corpo atual?", "Qual figura representa o corpo que você gostaria de ter?" e "Qual figura representa o corpo ideal?". Estes questionamentos geraram respectivamente as seguintes variáveis: "Silhueta atual"; "Silhueta desejada"; "Silhueta ideal".

O projeto foi encaminhado para apreciação de Comitê de Ética em Pesquisa, conforme resolução 196/96 do CNS e após sua aprovação foi iniciada a pesquisa, sendo critério de inclusão na amostra a aceitação voluntária do sujeito em participar do estudo demonstrado por meio da assinatura do Termo de Consentimento Livre Esclarecido, permanecendo uma cópia deste documento junto ao participante.

Após a assinatura do Termo de Consentimento Livre e Esclarecido, foi entregue aos participantes da pesquisa a ficha com dados sociodemográficos referente ao perfil de uso de substâncias psicoativas. Nos pacientes que não preenchiam nenhum critério de exclusão, foi realizada, individualmente, a avaliação antropométrica, e aplicada a escala de silhuetas.

Os dados foram analisados através do software Statistical Package for the Social Sciences (SPSS), versão 17.0, for windows. A análise dos dados contou de testes Descritivos e de Frequências e o teste Qui-Quadrado. O nível de significância utilizada como parâmetro foi de $5 \%$.

\section{RESULTADOS}

\section{CARACTERIZAÇÃO DA AMOSTRA}

A amostra foi composta por 100 sujeitos do sexo masculino, internados em unidade de desintoxicação hospitalar, em média, há 3,97 dias (DP=2,40;1-8) quando do momento de coleta dos dados, e tendo uma média de idade de 28,01 anos (DP=6,71; 18-45). Do total da amostra, 87\% ( $n=87)$ declararam-se solteiros, 9\% $(n=9)$ casados e $4 \%(n=4)$ separados. Também foi observada uma média de 7,82 anos de estudo $(\mathrm{DP}=2,60 ; 1-14)$, dado que equivaleria ao ensino fundamental incompleto a partir do modelo de educação brasileiro vigente. 


\section{PADRÃO DE CONSUMO}

O padrão de consumo de substâncias psicoativas foi questionado quanto ao seu uso, por tipo de substância em relação à quantidade, e último uso. Para melhor visualização, seus dados são descritos nas Tabelas 1 e 2.

Tabela 1. Uso na vida e último uso de substâncias psicoativas entre dependentes de crack hospitalizados para desintoxicação. Porto Alegre, RS, 2012

\begin{tabular}{|c|c|c|c|c|c|c|}
\hline \multirow{2}{*}{ Substância } & \multirow{2}{*}{$\begin{array}{l}\text { Uso na } \\
\text { vida }\end{array}$} & \multicolumn{5}{|c|}{ Último uso em dias } \\
\hline & & Até 7 & Até 30 & Até 90 & Até 360 & \begin{tabular}{|c} 
Mais de \\
360
\end{tabular} \\
\hline Álcool & $\begin{array}{c}99 \% \\
(\mathrm{n}=99)\end{array}$ & $\begin{array}{c}39,8 \% \\
(\mathrm{n}=39)\end{array}$ & $\begin{array}{c}31,6 \% \\
(\mathrm{n}=31)\end{array}$ & $\begin{array}{c}13,3 \% \\
(\mathrm{n}=13)\end{array}$ & $\begin{array}{c}7,1 \% \\
(\mathrm{n}=7)\end{array}$ & $\begin{array}{c}8,2 \% \\
(\mathrm{n}=8)\end{array}$ \\
\hline Tabaco & $\begin{array}{c}95 \% \\
(\mathrm{n}=95)\end{array}$ & $\begin{array}{c}86,2 \% \\
(\mathrm{n}=81)\end{array}$ & $\begin{array}{c}10,6 \% \\
(\mathrm{n}=10)\end{array}$ & $\begin{array}{c}1,1 \% \\
(n=1)\end{array}$ & - & $\begin{array}{c}2,1 \% \\
(\mathrm{n}=2)\end{array}$ \\
\hline Maconha & $\begin{array}{c}95 \% \\
(\mathrm{n}=95)\end{array}$ & $\begin{array}{c}36,8 \% \\
(\mathrm{n}=35)\end{array}$ & $\begin{array}{c}23,2 \% \\
(\mathrm{n}=22)\end{array}$ & $\begin{array}{c}11,6 \% \\
(\mathrm{n}=11)\end{array}$ & $\begin{array}{c}8,4 \% \\
(\mathrm{n}=8)\end{array}$ & $\begin{array}{c}20 \% \\
(\mathrm{n}=19)\end{array}$ \\
\hline Solvente & $\begin{array}{c}58 \% \\
(\mathrm{n}=58)\end{array}$ & $\begin{array}{c}1,8 \% \\
(\mathrm{n}=1)\end{array}$ & $\begin{array}{c}1,8 \% \\
(\mathrm{n}=1)\end{array}$ & $\begin{array}{c}5,3 \% \\
(\mathrm{n}=3)\end{array}$ & $\begin{array}{c}7 \% \\
(n=4)\end{array}$ & $\begin{array}{l}84,2 \% \\
(\mathrm{n}=48)\end{array}$ \\
\hline Cocaína Aspirada & $\begin{array}{c}89 \% \\
(\mathrm{n}=89)\end{array}$ & $\begin{array}{c}13 \% \\
(\mathrm{n}=12)\end{array}$ & $\begin{array}{c}23,9 \% \\
(\mathrm{n}=22)\end{array}$ & $\begin{array}{c}12 \% \\
(\mathrm{n}=11)\end{array}$ & $\begin{array}{c}12 \% \\
(\mathrm{n}=11)\end{array}$ & $\begin{array}{c}39,1 \% \\
(\mathrm{n}=36)\end{array}$ \\
\hline Cocaína E.V. & $\begin{array}{c}9 \% \\
(\mathrm{n}=9)\end{array}$ & . & - & . & - & $\begin{array}{l}100 \% \\
(\mathrm{n}=7)\end{array}$ \\
\hline Extase & $\begin{array}{c}11 \% \\
(n=11)\end{array}$ & $\begin{array}{c}8,3 \% \\
(\mathrm{n}=1)\end{array}$ & - & - & - & $\begin{array}{c}91,7 \% \\
(\mathrm{n}=11)\end{array}$ \\
\hline Sedativo & $\begin{array}{c}3 \% \\
(n=3)\end{array}$ & $\begin{array}{l}33,3 \% \\
(n=1)\end{array}$ & - & - & - & $\begin{array}{l}66,7 \% \\
(n=2)\end{array}$ \\
\hline Anabolizante & $\begin{array}{c}2 \% \\
(n=2)\end{array}$ & - & - & - & - & $\begin{array}{l}100 \% \\
(n=2)\end{array}$ \\
\hline Tranquilizante & $\begin{array}{c}20 \% \\
(n=20)\end{array}$ & $\begin{array}{l}21,1 \% \\
(\mathrm{n}=4)\end{array}$ & $\begin{array}{l}15,8 \% \\
(\mathrm{n}=3)\end{array}$ & $\begin{array}{c}5,3 \% \\
(\mathrm{n}=1)\end{array}$ & $\begin{array}{l}10,5 \% \\
(n=2)\end{array}$ & $\begin{array}{l}47,4 \% \\
(n=9)\end{array}$ \\
\hline Anfetamina & $\begin{array}{c}12 \% \\
(n=12)\end{array}$ & - & $\begin{array}{c}9,1 \% \\
(n=1)\end{array}$ & $\begin{array}{c}9,1 \% \\
(\mathrm{n}=1)\end{array}$ & - & $\begin{array}{l}81,8 \% \\
(n=9)\end{array}$ \\
\hline Crack & $\begin{array}{c}100 \% \\
(n=100)\end{array}$ & $\begin{array}{c}69,7 \% \\
(\mathrm{n}=69)\end{array}$ & $\begin{array}{c}29,3 \% \\
(\mathrm{n}=29)\end{array}$ & $\begin{array}{c}1 \% \\
(n=1)\end{array}$ & . & - \\
\hline Lsd & $\begin{array}{c}8 \% \\
(n=8)\end{array}$ & $\begin{array}{l}11,1 \% \\
(n=1)\end{array}$ & - & $\begin{array}{l}33,3 \% \\
(\mathrm{n}=3)\end{array}$ & $\begin{array}{l}11,1 \% \\
(n=1)\end{array}$ & $\begin{array}{l}44,4 \% \\
(n=4)\end{array}$ \\
\hline Heroína & $\begin{array}{c}2 \% \\
(\mathrm{n}=2)\end{array}$ & - & - & . & - & $\begin{array}{l}100 \% \\
(\mathrm{n}=1)\end{array}$ \\
\hline Alucinógenos & $\begin{array}{c}11 \% \\
(n=11)\end{array}$ & - & $\begin{array}{c}9,1 \% \\
(\mathrm{n}=1)\end{array}$ & - & - & $\begin{array}{c}90,9 \% \\
(\mathrm{n}=10)\end{array}$ \\
\hline Oxi & $\begin{array}{c}10 \% \\
(n=10)\end{array}$ & $\begin{array}{l}11,1 \% \\
(n=1)\end{array}$ & $\begin{array}{l}44,4 \% \\
(n=4)\end{array}$ & $\begin{array}{l}11,1 \% \\
(\mathrm{n}=1)\end{array}$ & $\begin{array}{l}33,3 \% \\
(n=3)\end{array}$ & - \\
\hline
\end{tabular}

A variável "uso na vida" diz respeito ao percentual da amostra que já fez uso em pelo menos um momento de determinada substância, independentemente do seguimento do uso ou não. Enquanto que o "Último uso" refere-se ao tempo decorrido do último episódio de utilização da substância (por menor que fosse este uso) até o dia da coleta dos dados, ou seja, o tempo de abstinência da população que realizou o uso de determinada substância. As categorias "Sedativo", "Anabolizante", "Tranquilizante", e "Anfetamina" dizem respeito à utilização sem prescrição médica.

Tabela 2. Idade de início do uso e quantidade média de substâncias psicoativas utilizadas por dependentes de crack hospitalizados para desintoxicação. Porto Alegre, RS, 2012

(Continua)

\begin{tabular}{|c|c|c|c|c|}
\hline \multirow{2}{*}{ Substância } & \multicolumn{2}{|c|}{ Idade de início } & \multicolumn{2}{|c|}{$\begin{array}{l}\text { Quantidade média uti- } \\
\text { lizada }\end{array}$} \\
\hline & $\begin{array}{l}\text { Média } \\
\text { (DP) }\end{array}$ & $\begin{array}{l}\text { Mín.- } \\
\text { Máx. }\end{array}$ & Média (DP) & Mín.- Máx. \\
\hline Álcool & $\begin{array}{l}12,90 \\
(3,68) \\
\end{array}$ & $1-22$ & $\begin{array}{c}827,81 \\
(1616,74) \\
\end{array}$ & $\begin{array}{c}0,05- \\
7466,67\end{array}$ \\
\hline Tabaco & $\begin{array}{l}14,22 \\
(3,65) \\
\end{array}$ & $6-33$ & $\begin{array}{c}209,88 \\
(146,71) \\
\end{array}$ & $\begin{array}{c}7,00- \\
900,00\end{array}$ \\
\hline Maconha & $\begin{array}{l}14,72 \\
(3,10) \\
\end{array}$ & $9-32$ & $14,46(15,64)$ & $0,25-70,00$ \\
\hline Solvente & $\begin{array}{l}15,91 \\
(3,56) \\
\end{array}$ & $10-28$ & $100,00(-)$ & - \\
\hline $\begin{array}{l}\text { Cocaína Aspi- } \\
\text { rada }\end{array}$ & $\begin{array}{l}17,35 \\
(3,99) \\
\end{array}$ & $11-35$ & $1,81(1,96)$ & $0,2-7,00$ \\
\hline Cocaína E.V. & $\begin{array}{l}17,56 \\
(3,43) \\
\end{array}$ & $13-23$ & - & - \\
\hline Extase & $\begin{array}{l}20,73 \\
(4,62) \\
\end{array}$ & $17-31$ & $0,50(-)$ & - \\
\hline Sedativo & $\begin{array}{l}20,00 \\
(2,64) \\
\end{array}$ & $18-23$ & - & - \\
\hline Anabolizante & $\begin{array}{l}17,50 \\
(2,12) \\
\end{array}$ & $16-19$ & - & - \\
\hline Tranquilizante & $\begin{array}{l}18,45 \\
(4,86) \\
\end{array}$ & $12-30$ & $11,83(8,37)$ & $2,00-21,00$ \\
\hline Anfetamina & $\begin{array}{l}21,25 \\
(6,56) \\
\end{array}$ & $12-35$ & - & - \\
\hline Crack & $\begin{array}{l}21,08 \\
(6,72)\end{array}$ & $2-37$ & $14,30(17,12)$ & $0,5-100,00$ \\
\hline LSD & $\begin{array}{l}22,75 \\
(7,28) \\
\end{array}$ & $16-37$ & $0,50(-)$ & - \\
\hline Heroína & $\begin{array}{l}21,50 \\
(7,77) \\
\end{array}$ & $16-27$ & - & - \\
\hline Alucinógenos & $\begin{array}{l}17,00 \\
(2,09) \\
\end{array}$ & $15-21$ & - & - \\
\hline
\end{tabular}


(Conclusão) Tabela 3. Frequência de autoimagem corporal a partir da

\begin{tabular}{l|c|c|c|c}
\hline \multirow{2}{*}{ Substância } & \multicolumn{2}{|c|}{ Idade de início } & \multicolumn{2}{|c}{$\begin{array}{c}\text { Quantidade média uti- } \\
\text { lizada }\end{array}$} \\
\cline { 2 - 5 } & $\begin{array}{c}\text { Média } \\
\text { (DP) }\end{array}$ & $\begin{array}{c}\text { Mín.- } \\
\text { Máx. }\end{array}$ & Média (DP) & Mín.- Máx. \\
\hline Oxi & $\begin{array}{c}26,20 \\
(5,73)\end{array}$ & $20-36$ & $1,12(1,25)$ & $0,5-30,00$ \\
\hline
\end{tabular}

Utilizou-se para mensuração da quantidade média utilizada por semana no último mês, as seguintes unidades de medida: Unidades Internacionais/ UI de álcool (1 UI equivale a 10 gramas de álcool); cigarros (tabaco); baseados (maconha); comprimidos (anabolizante, tranquilizante, sedativo, anfetamina); mililitros (solvente e alucinógeno).

\section{COMPOSIÇÃO CORPORAL}

Observou-se um peso médio de $70,56 \mathrm{~kg}$ $(\mathrm{DP}=12,76 ; 50-127,30)$ e estatura média de $1,71 \mathrm{~m}$ ( $\mathrm{DP}=0,05 ; 1,55-1,86)$, o que gerou um IMC médio de $24,01 \mathrm{~kg} / \mathrm{m}^{2}(\mathrm{DP}=3,65 ; 17,30-39,29)$. Referente à mensuração dos perímetros, encontrou-se uma média de 85,02 $\mathrm{cm}$ para a cintura $(\mathrm{DP}=9,16 ; 68,30-124,3)$ e $96,22 \mathrm{~cm}$ para o quadril (DP=7,44; 83,10-126,10), gerando uma relação cintura-quadril (RCQ) média de $0,87(\mathrm{DP}=0,10$; 0,08-1,04). Para ambos os cálculos foram utilizadas as fórmulas preconizadas pela Organização Mundial da Saúde (1995).

\section{AUTOIMAGEM}

Por meio da análise descritiva, mais precisamente da média, foi possível identificar que a imagem corporal atual apontada pelos indivíduos foi representada pela Figura 4 da escala das Silhuetas com IMC entre $18,75 \mathrm{~kg} /$ $\mathrm{m}^{2}$ e $21,24 \mathrm{~kg} / \mathrm{m}^{2}$, todavia a silhueta real foi a da Figura $5 \mathrm{da}$ escala das Silhuetas que possui IMC entre $21,25 \mathrm{~kg} /$ $\mathrm{m}^{2}$ e $23,74 \mathrm{~kg} / \mathrm{m}^{2}$, enquanto que a silhueta desejada e a silhueta ideal foram representadas pela Figura 6 da escala das Silhuetas de IMC entre $23,75 \mathrm{~kg} / \mathrm{m}^{2}$ e $26,24 \mathrm{~kg} / \mathrm{m}^{2}$.

Na Tabela 3, está apresentada a frequência de autoimagem corporal dos pacientes a partir da distribuição das cartas correspondentes às silhuetas real, atual desejada e ideal questionadas aos sujeitos. escolha das silhuetas em dependentes de crack hospitalizados para desintoxicação. Porto Alegre, RS, 2012

\begin{tabular}{|c|c|c|c|c|}
\hline $\begin{array}{c}\text { Escala de silhueta } \\
\text { (intervalo de } \\
\text { IMC*) }\end{array}$ & $\begin{array}{c}\text { Silhueta } \\
\text { real \% (n) }\end{array}$ & $\begin{array}{l}\text { Silhueta } \\
\text { atual \% } \\
\text { (n) }\end{array}$ & $\begin{array}{c}\text { Silhueta } \\
\text { desejada \% } \\
\text { (n) }\end{array}$ & $\begin{array}{c}\text { Silhueta } \\
\text { ideal \% } \\
\text { (n) }\end{array}$ \\
\hline $1(11,25$ a 13,74$)$ & - & $\begin{array}{c}14,0 \% \\
(14) \\
\end{array}$ & - & - \\
\hline $2(13,75$ a 16,24$)$ & - & $\begin{array}{c}14,0 \% \\
(14) \\
\end{array}$ & $2,0 \%(2)$ & $1,0 \%(1)$ \\
\hline $3(16,25$ a 18,74$)$ & $4,0 \%(4)$ & $\begin{array}{c}22,0 \% \\
(22) \\
\end{array}$ & $2,0 \%(2)$ & $2,0 \%(2)$ \\
\hline $4(18,75$ a 21,24$)$ & $\begin{array}{c}17,0 \% \\
(17) \\
\end{array}$ & $\begin{array}{c}14,0 \% \\
(14) \\
\end{array}$ & $12,0 \%(12)$ & $\begin{array}{c}15,0 \% \\
(15) \\
\end{array}$ \\
\hline $5(21,25$ a 23,74$)$ & $\begin{array}{c}38,0 \% \\
(38) \\
\end{array}$ & $\begin{array}{c}12,0 \% \\
(12) \\
\end{array}$ & $18,0 \%(18)$ & $\begin{array}{c}13,0 \% \\
(13) \\
\end{array}$ \\
\hline $6(23,75$ a 26,24$)$ & $\begin{array}{c}16,0 \% \\
(16)\end{array}$ & $7,0 \%(7)$ & $30,0 \%(30)$ & $\begin{array}{c}28,0 \% \\
(28)\end{array}$ \\
\hline $7(26,25$ a 28,74$)$ & $\begin{array}{c}12,0 \% \\
(12)\end{array}$ & $6,0 \%(6)$ & $16,0 \%(16)$ & $\begin{array}{c}19,0 \% \\
(19)\end{array}$ \\
\hline $8(28,75$ a 31,24$)$ & $7,0 \%(7)$ & $6,0 \%(6)$ & $7,0 \%(7)$ & $\begin{array}{c}11,0 \% \\
(11)\end{array}$ \\
\hline $9(31,25$ a 33,74$)$ & $2,0 \%(2)$ & $1,0 \%(1)$ & $9,0 \%(9)$ & $8,0 \%(8)$ \\
\hline $\begin{array}{l}10(33,75 \text { a } \\
36,24)\end{array}$ & $1,0 \%(1)$ & $3,0 \%(3)$ & $3,0 \%(3)$ & $2,0 \%(2)$ \\
\hline $\begin{array}{l}11(36,25 \mathrm{a} \\
38,74)\end{array}$ & - & $1,0 \%(1)$ & - & - \\
\hline $\begin{array}{l}12(38,75 \mathrm{a} \\
41,24)\end{array}$ & $1,0 \%(1)$ & - & $1,0 \%(1)$ & - \\
\hline $\begin{array}{l}13(41,25 \mathrm{a} \\
43,74) \\
\end{array}$ & - & - & - & $\cdot$ \\
\hline $\begin{array}{l}14(43,75 \mathrm{a} \\
46,24)\end{array}$ & - & - & - & - \\
\hline $\begin{array}{l}15(46,25 \mathrm{a} \\
48,75)\end{array}$ & - & - & - & $1,0 \%(1)$ \\
\hline
\end{tabular}

*Os valores de IMC são descritos utilizando-se a seguinte unidade de medida: $\mathrm{kg} / \mathrm{m}^{2}$.

Também foi computado, segundo protocolo de avaliação do instrumento, variável que descreve a distorção da imagem corporal (diferença entre IMC atual e IMC real) e variável que descreve a insatisfação da imagem corporal (diferença entre IMC desejado e IMC atual) correspondentes às figuras apontadas, o que demonstrou uma média de distorção igual a -1,48 (DP=1,80; -6-3), e insatisfação média igual a 2,13 (DP=2,85; -6-8).

Foi encontrada, por meio do teste de Qui-Quadrado, diferença significativa entre a autoimagem da silhueta real (estimada por intermédio do IMC) e a silhueta atual $\left({ }^{2}=153,25 ; \mathrm{p}<0,001\right)$. Não foi encontrada asso- 
ciação significativa entre a silhueta real e a silhueta ideal $\left({ }^{2}=69,47 ; \mathrm{p}=0,563\right)$ e desejada $\left({ }^{2}=74,45 ; \mathrm{p}=0,397\right)$. A silhueta real era correspondente, para 59\% da amostra, às silhuetas de 3 a 5 , a silhueta atual, para $64 \%$, equivalia às silhuetas de $1 \mathrm{a} 4$, a silhueta desejada era, para $75 \%$, semelhante às silhuetas de $4 \mathrm{a} 7 \mathrm{e}$ a silhueta ideal correspondeu, para $71 \%$, às silhuetas de 5 a 8 . Assim, não houve diferenças significativas entre a silhueta real e tanto a atual, mas os pacientes apontaram imagens de silhuetas desejada e ideal com um IMC maior do que eles apresentam atualmente.

\section{DISCUSSÃO}

A presente amostra demonstrou padrão sociodemográfico semelhante ao apresentado por outras pesquisas que abordaram dependentes de crack, ou seja, adultos jovens, sendo predominantemente composta por sujeitos solteiros e com baixa escolaridade. ${ }^{16,21-23}$

Observou-se que os valores antropométricos encontrados ficaram próximos aos descritos previamente em estudo realizado no sul do Brasil ${ }^{16}$ com amostra composta por dependentes de crack, no qual foi encontrado IMC médio de 23,79 ( $\mathrm{DP}=4,39 ; 18,09-39,24)$, valor próximo ao encontrado na presente pesquisa, que foi de $24,01 \mathrm{~kg} / \mathrm{m}^{2}$ (DP=3,65; 17,30-39,29). Assim como a relação (RCQ) média, que no presente estudo foi de 0,87 $(\mathrm{DP}=0,10 ; 0,08-1,04)$, enquanto que no estudo citado foi de $0,86(\mathrm{DP}=0,04 ; 0,75-0,98)$.

Referente à imagem corporal, foi possível identificar que os dependentes de crack da amostra apresentam diferença estatisticamente significante entre a silhueta real e as silhuetas apontadas como desejada e ideal pelos mesmos, corroborando a literatura, que afirma a distorção da imagem entre os sujeitos (tanto homens como mulheres). ${ }^{24,25}$ Pelos resultados, também foi possível identificar um desejo dos indivíduos em aumentar o tamanho de seu corpo. Deste modo, a presente amostra difere dos resultados da pesquisa realizada com amostra composta por homens universitários não usuários de substâncias psicoativas que, inversamente a presente pesquisa, apresentou IMC real acima do valor de IMC desejado. ${ }^{8}$

Também é possível identificar por meio da distorção da imagem corporal, que os indivíduos fazem su- bestimação de seu tamanho corporal. Aspecto que se corroboram dados descritos na literatura científica ao relatar que os homens apresentam tendência em subestimar o tamanho corporal. ${ }^{8}$

A subestimação da imagem corporal dos integrantes da amostra reflete aspectos intrínsecos da cultura popular, pois há uma imagem do dependente de crack fora dos padrões estéticos e muitas vezes padrões de saúde, com peso abaixo do que é considerado saudável pelo senso comum. ${ }^{26}$ Esta ideia é apontada pelo indivíduo da pesquisa ao demonstrar desejo em aumentar o tamanho corporal.

Esse desejo em aumentar o tamanho corporal, na busca de um melhor enquadramento estético é um importante aspecto a ser utilizado como fator de motivação para início e manutenção do tratamento e da abstinência frente ao uso. ${ }^{1,13}$ Também há a importância da elaboração de um trabalho de psicoeducação referente ao aspecto anorexígeno da utilização de crack, gerando uma conscientização acerca de mais um aspecto danoso do abuso e da dependência da cocaína/crack. ${ }^{27}$

O desenvolvimento de um trabalho corporal que almeja uma aproximação dos valores antropométricos à representatividade corporal imaginada e desejada pelo indivíduo é relevante, pois a insatisfação com o próprio corpo tende a ser uma situação de risco para recaída. ${ }^{23}$ Todavia, a busca pelo aumento do tamanho corporal deve ser acompanhada por profissional competente, pois como apontado pela literatura ${ }^{21}$, o aumento do tamanho corporal, muitas vezes, restringe-se ao aumento da quantidade de gordura corporal, sem modificação significativa da massa muscular, o que tende a favorecer ao surgimento e/ou agravamento principalmente de patologias metabólicas e cardiovasculares. ${ }^{28}$

Pode-se observar como uma das limitações do estudo o tipo de delineamento metodológico utilizado, pois caso fossem investigadas associações entre variáveis da autoimagem em relação às variáveis de consumo de crack com o intuito de verificar causalidade, tenderia existir um viés causalidade reversa. Uma abordagem longitudinal poderia contribuir substancialmente para elucidação dos fenômenos que permeiam o tema. Também seria valioso analisar as diferentes medicações utilizadas durante o tratamento e a alimentação. 


\section{CONCLUSÃO}

Fica evidente por meio deste estudo que o dependente de crack possui desejo de aumentar seu tamanho corporal. Esta insatisfação com a imagem corporal pode estar relacionada com a perda de peso proveniente da falta de alimentação durante o período de consumo da droga, assim como ao rótulo culturalmente empregado aos dependentes de crack associado a uma figura com baixo peso. O sofrimento e desconforto gerado pela insatisfação com a imagem corporal pode ser um aspecto potencializador do desencadeamento de lapso e, mais gravemente, de recaída por parte destes indivíduos.

Cabe frisar que os dados demonstram relevância, pois podem (e devem) ser utilizados pelos profissionais da área da saúde para repensar a prática e, consequentemente, desenvolver melhorias no atendimento ao dependente de crack, abrangendo, dentro de seu projeto terapêutico individualizado, o emprego de atividade física e do trabalho da imagem corporal dos sujeitos. Esta modificação pode culminar em menor nível de insatisfação com o próprio corpo e assim aumentar a possibilidade da manutenção de abstinência dos sujeitos.

\section{REFERÊNCIAS}

1. Hughes EK, Gullone E. Emotion regulation moderates relationships between body image concerns and psychological symptomatology. Body Image. 2011;8(3):224-31.

2. Kakeshita IS, Silva AIP, Zanatta DP, Almeida SS. Construção e fidedignidade teste-reteste de escalas de silhuetas brasileiras para adultos e crianças. Psic.: Teor. e Pesq. 2009;25(2):263-70.

3. Saviola MG. A imagem corporal. Rev. Bras. Psiquiatr. 2003;25(2): 126-126.

4. Skopinski F, Resende TL, Schneider RH. Imagem corporal, humor e qualidade de vida. Revista Brasileira de Geriatria e Gerontologia. 2015; 18(1): 95-105.

5. Alvarenga MS, Philippi ST, Lourenço BH, Sato PM, Scagliusi FB. Insatisfação com a imagem corporal em universitárias brasileiras. J. bras. psiquiatr. 2010;
59(1):44-51.

6. Morgano FFR, Ferreira MEC, Alexandrino DFL, Amaral ACS. Escala de silhuetas bidimensionais para avaliação da satisfação corporal do cego. Anais da XVI CONBRACE e III CONICE. 2009; 1-10, Salvador, Brasil. CONBRACE/CONICE; 2009.

7. Barros DD. Imagem corporal: a descoberta de si mesmo. Hist. cienc. saude-Manguinhos, 2005;12(2):54754.

8. Kakeshita IS, Almeida SS. Relação entre índice de massa corporal e a percepção da autoimagem em universitários. Revista Saúde Pública. 2006;40(3):497-504.

9. Damasceno VO, Lima JRP, Vianna JM, Vianna VRA, Novaes JS. Tipo físico ideal e satisfação com a imagem corporal de praticantes de caminhada. Rev Bras Med Esp. 2005;11(3):181-6.

10. Tylka TL. Refinement of the tripartite influence model for men: Dual body image pathways to body change behaviors. Body Image. 2011;8(3):199- 207.

11. Silva DAS, Nahas MV, Sousa TF, Del Duca GF, Peres KG. Prevalence and associated factors with body image dissatisfaction among adults in southern Brazil: a population-based study. Body Image. 2011; 8(4):427-31.

12. Klos LA, Esser VE, Kessler MM. To weigh or not to weigh: the relationship between self-weighing behavior and body image among adults. Body Image. 2012; 9(4): 551-4.

13. Blashill AJ. Elements of male body image: Prediction of depression, eating pathology and social sensitivity among gay men. Body Image. 2010; 7(4): 310-6.

14. Ochoa ACG. Reflexiones sobre el tratamiento de transtornos paranoides inucidos pconsumo de cocaína. Liberaddictus. 2006; 90: 3-8.

15. Lizasoain I, Moro MA, Lorenzo P. Cocaína: aspectos farmacológicos. Adicciones. 2002; 14(1):37-45.

16. Balbinot AD, Alves GSL, Junior AFA, Araujo RB. Perfil antropométrico de dependentes de crack hospi- 
talizados para desintoxicação. Revista HCPA. 2011; 31(3): 311-317.

17. Sichieri R, Moura EC. Análise multinível das variações no índice de massa corporal entre adultos. Rev. Saúde Pública. 2006; 43(2): 90-7.

18. Langoni POO, Aerts DRGC, Alves GG, Câmara SG. Insatisfação com a imagem corporal e fatores associados em adolescentes escolares. Diaphora. 2014; 12(1): 23-30.

19. Dobmeyer AC, Peterson AL, Runyan CR, Hunter CM, Blackman LR. Body image and tobacco cessation: relationships with weight concerns and intention to resume tobacco use. Body Image. 2005; 2(2):187-92.

20. SENGIK AS, SCORTEGAGNA, SA. Consumo de drogas psicoativas em adolescentes escolares. Psic: Rev da Vetor Editora. 2008; 9(1): 73-80.

21. Balbinot AD, Alves GS, Junior AFA, Araujo RB. Associação entre fissura e perfil antropométrico em dependentes de crack. Online Jornal Bras Psiq. 2011; 60(3).

22. Oliveira LG, Nappo SA. Crack-cocaine in São Paulo: accessibility, market strategies and ways of use. Rev Psiq Clín. 2008; 35(6): 212-8.

23. Araujo RB, Pansard M, Boeira BU, Rocha NS. As estratégias de coping para o manejo da fissura de dependentes de crack. Revista HCPA. 2010; 30(1): 36-42.

24. Silva RS, Ferreira VL. Avaliação da auto-imagem em frequentadores de academia do interior do Rio Grande do Sul. Encontro: Revista de psicologia. 2013;16(24):23-30

25. Pope HG, Gruber AJ, Mangweth B, Decol C, Jouvent $\mathrm{R}$, Hudson JI. Body image perception among men in three countries. Psychiatry. 2000;157(8):1297-1301.

26. Almeida, FDJF. Perfil Antropométrico de Dependentes Químicos em Tratamento na Casa de AcolhidaChácara São Miguel Arcanjo. Revista Ceuma Perspectivas. 2017; 28(2), 18-25.

27. Pinto ACS, Beserra EP, Luna IT, Bezerra, LLDAL, Pi- nheiro PNDC. Educational practice with young crack users aiming the prevention of HIV/AIDS. Escola Anna Nery.2016; 20(3).

28. Massaroli LC, Santos LC, Carvalho GG, Carneiro SAJ, Rezende LF. Qualidade de vida e o imc alto como fator de risco para doenças cardiovasculares: revisão sistemática. Revista da Universidade Vale do Rio Verde. $2018 ; 16(1): 1-10$.

Recebido em: 09/09/2017

Aceito em: 01/03/2018 